\title{
COMPLETION OF PROBABILISTIC NORMED SPACES
}

\author{
BERNARDO LAFUERZA GUILLÉN \\ JOSÉ ANTONIO RODRÍGUEZ LALLENA \\ Departamento de Matemática Aplicada \\ Universidad de Almeria \\ 04120 Almeria, Spain \\ and \\ CARLO SEMPI \\ Dipartimento di Matematica \\ Universitá di Lecce \\ 73100 Lecce, Italy
}

(Received December 6, 1993 and in revised form January 28, 1994)

\begin{abstract}
We prove that every probabilistic normed space, either according to the original definition given by Šstnev, or according to the recent one introduced by Alsina, Schweizer and Sklar, has a completion.
\end{abstract}

KEY WORDS AND PHRASES. Probabilistic Normed Spaces, completion, triangle function, Lévy distance.

1991 AMS SUBJECT CLASSIFICATION CODES. 54E70, 60E99.

\section{INTRODUCTION.}

As is well known, a real or complex normed linear space admits a completion, namely, given a normed linear space $(V,\|\cdot\|)$, there exists another linear space $\left(V^{\prime},\|\cdot\|^{\prime}\right)$ such that $V^{\prime}$ is isometric to a dense subspace of $V$.

It was proved by Muštari [2], Sherwood ([7], [8]) and Sempi [5] that a probabilistic metric space has a completion. Here we answer in the positive the natural question of whether a probabilistic normed space has a completion. In fact, there are two definitions of probabilistic normed space ( = $P N$-space): the original one by Šerstnev ([6], but see [3] for a presentation in agreement with our notation), and a more recent one by Alsina, Schweizer and Sklar (see [1]). The proof will be given in both cases. For the notation and the concepts used we refer to the book by Schweizer and Sklar [3]; we shall write d.f. for distribution function.

According to Šerstnev, a $P N$-space is a triple $(V, v, \tau)$, where $V$ is a real linear space; $\tau$ is a triangle function ([3], section 7.1), i.e., a binary operation on $\Delta^{+}$, the space of distance distribution functions, that is commutative, associative and nondecreasing in each variable and which has the d.f. $\varepsilon_{0}$ as identity, i.e.,

(a) $\forall F, G \in \Delta^{+} \quad \tau(F, G)=\tau(G, F)$;

(b) $\forall F, G, H \in \Delta^{+} \quad \tau(F, \tau(G, H)=\tau(\tau(F, G), H)$;

(c) $\forall H \in \Delta^{+} F \leq G \Rightarrow \quad \tau(F, H) \leq \tau(G . H)$;

(d) $\forall F \in \Delta^{+} \quad \tau\left(F, \varepsilon_{0}\right)=F$.

Here $\varepsilon_{0}$ is the d.f. defined by

$$
\varepsilon_{0}(x):= \begin{cases}0, & \text { if } x \leq 0 \\ 1, & \text { if } x>0\end{cases}
$$


$\nu$ is the probabilistic norm, i.e., $\nu$ is a 111, from $V$ into $\Delta^{+}$that satisfies the following conditions:

(N.1) $\nu(p)=\varepsilon_{0}$, if, and only if, $p=\vartheta$, where $\vartheta$ is the null vector of $V$;

(N.2) $\forall x \in \mathbb{R}_{+}, a \in \mathbb{R}$, with $a \neq 0 \quad \nu(a p)(x)=\nu(p)(x /|a|)$;

(N.3) $\forall p, q \in V \quad \nu(p+q) \geq \tau[\nu(p), \nu(q)]$.

In both definitions the triangle function is assumed to be continuous.

The space $\Delta^{+}$can be metrized by different metrics ([9], [3], [4], [10]), but we shall use here the modified Lévy metric $d_{L}[3]$.

\section{MAIN RESULTS.}

THEOREM 1. Every $P N$-space $(V, \nu, \tau)$ has a completion, viz. is isometric to a dense linear subspace of a complete $P N$-space $\left(V^{\prime}, \nu^{\prime}, \tau\right)$.

PROOF. Only the steps needed to complement the treatment in [7] and [8] will be given. Now $V^{\prime}$ is the set of equivalence classes of Cauchy sequences of elements of $V$. In order to prove that $V^{\prime}$ is a linear space, let $p^{\prime}$ and $q^{\prime}$ be elements of $V^{\prime}$ and let $\left\{p_{n}\right\}$ and $\left\{q_{n}\right\}$ be Cauchy sequences of elements of $V$ with $\left\{p_{n}\right\} \in p^{\prime}$ and $\left\{q_{n}\right\} \in q^{\prime}$. Since $V$ is a linear space, one has, for every $n \in \mathbb{N}, p_{n}+q_{n} \in V$. We wish to show that it is possible to define a sum of $p^{\prime}$ and $q^{\prime}$ in such a way that $p^{\prime}+q^{\prime} \in V^{\prime}$. Since $(V, \mathcal{F}, \tau)$, with $\mathscr{F}(p, q):=\nu(p-q)$ is a probabilistic metric space ([3], Theorem 15.1.2), one has, if $n$ and $m$ are large enough,

$$
\begin{gathered}
\mathcal{F}\left(p_{n}+q_{n}, p_{m}+q_{m}\right)=\nu\left(\left(p_{n}+q_{n}\right)-\left(p_{m}+q_{m}\right)\right) \\
\left.=\nu\left(\left(p_{n}-p_{m}\right)+\left(q_{n}-q_{m}\right)\right) \quad \text { (because of }(\mathrm{N} .3)\right) \\
\geq \tau\left[\nu\left(p_{n}-p_{m}\right), \nu\left(q_{n}-q_{m}\right)\right] .
\end{gathered}
$$

Taking into account Lemma 4.3.4 in [3], one has

$$
\begin{gathered}
d_{L}\left(\mathcal{F}\left(p_{n}+q_{n}, p_{m}+q_{m}\right), \varepsilon_{0}\right) \leq d_{L}\left(\tau\left[\nu\left(p_{n}-p_{m}\right), \nu\left(q_{n}-q_{m}\right)\right], \varepsilon_{0}\right) \\
=d_{L}\left(\tau\left[\mathcal{F}\left(p_{n}, p_{m}\right), \mathcal{F}\left(q_{n}, q_{m}\right)\right], \varepsilon_{0}\right) .
\end{gathered}
$$

The continuity of both $d_{L}$ and $\tau$ ensures that, when both $m$ and $n$ tend to infinity, $\mathscr{F}\left(p_{n}+q_{n}, p_{m}+q_{m}\right) \stackrel{w}{\rightarrow} \varepsilon_{0}$. Thus $\left\{p_{n}+q_{n}\right\}$ is a Cauchy sequence and, as a consequence, it belongs to an element of $V^{\prime}$, which will be denoted by $r^{\prime}$. Then we define $p^{\prime}+q^{\prime}=r^{\prime}$. This definition does not depend on the elements of $p^{\prime}$ and $q^{\prime}$ selected, for, if $\left\{p_{n}\right\},\left\{p_{n}^{*}\right\} \in p^{\prime}$ and $\left\{q_{n}\right\},\left\{q_{n}^{*}\right\} \in q^{\prime}$, then

$$
\mathscr{F}\left(p_{n}+q_{n}, p_{n}^{*}+q_{n}^{*}\right)=\nu\left(p_{n}-p_{n}^{*}, q_{n}-q_{n}^{*}\right) \geq \tau\left[\nu\left(p_{n}-p_{n}^{*}\right), \nu\left(q_{n}-q_{n}^{*}\right)\right]=\tau\left[\mathcal{F}\left(p_{n}, p_{n}^{*}\right), \mathcal{F}\left(q_{n}, q_{n}^{*}\right)\right]
$$

so that

$$
d_{L}\left(\mathcal{F}\left(p_{n}+q_{n}, p_{n}^{*}+q_{n}^{*}\right), \varepsilon_{0}\right) \leq d_{L}\left(\tau\left[\mathcal{F}\left(p_{n}, p_{n}^{*}\right), \mathscr{F}\left(q_{n}, q_{n}^{*}\right)\right], \varepsilon_{0}\right)
$$

Since both $d_{L}$ and $\tau$ are continuous we obtain $\mathscr{F}\left(p_{n}+q_{n}, p_{n}^{*}+q_{n}^{*}\right) \stackrel{w}{\rightarrow} \varepsilon_{0}$, i.e., $\left\{p_{n}+q_{n}\right\} \sim\left\{p_{n}^{*}+q_{n}^{*}\right\}$. Thus the sum defined above is a good definition, which immediately satisfies the properties of an abelian group.

For every $\alpha \in \mathbb{R}$, and for every Cauchy sequence $\left\{p_{n}\right\}$ of elements of $V$, also $\left\{\alpha p_{n}\right\}$ is a Cauchy sequence of elements of $V$. This is obvious if $\alpha=0$. If $\alpha \neq 0$, one has, for every $x>0$,

$$
\begin{aligned}
\mathcal{F}\left(\alpha p_{n}, \alpha p_{m}\right)(x) & =\nu\left(\alpha p_{n}-\alpha p_{m}\right)(x)=\nu\left(p_{n}-p_{m}\right)(x /|\alpha|) \\
& =\mathscr{F}\left(p_{n}, p_{m}\right)(x /|\alpha|),
\end{aligned}
$$

and this tends to 1 , for every $x>0$, as $n$ and $m$ tend to infinity, i.e., $\mathscr{F}\left(\alpha p_{n}, \alpha p_{m}\right) \stackrel{w}{\rightarrow} \varepsilon_{0}$. Thus $\left\{\alpha p_{n}\right\}$ is a Cauchy sequence; let us denote by $u^{\prime}$ the element of $V^{\prime}$ to which it belongs. Then we define $\alpha p^{\prime}=u^{\prime}$. This is again a good definition; in fact, let $\left\{p_{n}\right\},\left\{p_{n}^{*}\right\} \in p^{\prime}$. Then

$$
\mathscr{F}\left(\alpha p_{n}, \alpha p_{n}^{*}\right)(x)=\nu\left[\alpha\left(p_{n}-p_{n}^{*}\right)\right](x)=\nu\left(p_{n}-p_{n}^{*}\right)\left(\frac{x}{|\alpha|}\right)=\mathscr{F}\left(p_{n}, p_{n}^{*}\right)\left(\frac{x}{|\alpha|}\right)
$$


which tends to 1 for all $x>0$ when $n \rightarrow \infty$, whence $\left\{\alpha p_{n}\right\} \sim\left\{\alpha p_{n}^{*}\right\}$. Therefore it is immediate to conclude that $V^{\prime}$ is a linear space. All that is left to show is that the distance d.f. $\mathcal{F}^{\prime}$ derives from a probabilistic norm $\nu^{\prime}$ on $V^{\prime}$. For every $p^{\prime} \in I^{\prime \prime}$, set, if $\left\{p_{n}\right\} \in p^{\prime}$ with $p_{n} \in V$ for every $n \in \mathbb{N}$

$$
v^{\prime}\left(p^{\prime}\right):=\mathcal{F}^{\prime}\left(p^{\prime}, \vartheta^{\prime}\right)=\lim _{n} \mathcal{F}\left(p_{n}, \vartheta\right)=\lim _{n} v\left(p_{n}\right)
$$

Thus

$$
\mathcal{F}^{\prime}\left(p^{\prime}, q^{\prime}\right)=\lim _{n} \mathcal{F}^{(}\left(p_{n}, q_{n}\right)=\lim _{n} \nu\left(p_{n}-q_{n}\right)=\nu^{\prime}\left(p^{\prime}-q^{\prime}\right)
$$

It is now an easy task to verify that $\nu^{\prime}$ does indeed fulfill conditions (N.1), (N.2) and (N.3).

We now turn to the proof of the analogous result for $P N$-spaces according to the definition given in [1]. This latter differs from the one given above in that condition (N.2) is replaced by the weaker one

$$
\forall p \in V \quad \nu(-p)=\nu(p)
$$

and a new one is added:

$$
\forall \alpha \in[0,1] \forall p \in V \quad \nu(p) \leq \tau^{*}[\nu(\alpha p), \nu((1-\alpha) p)] .
$$

Then a $P N$-space is a quadruple $\left(V, \nu, \tau, \tau^{*}\right)$, where $V$, as above, is a real linear space, $\tau, \tau^{*}$ are continuous triangle functions and $\nu^{\prime}: V \rightarrow \Delta^{+}$is a map such that conditions (N.1), (N.2'), (N.3) and (N.4) are satisfied.

The last part of this note is entirely devoted to $P N$-spaces according to this latter definition.

LEMMA 2. Let $\left(V, \nu, \tau, \tau^{*}\right)$ be a $P N$-space and let $h$ and $k$ be two real constants such that $0 \leq h \leq k$; then

$$
\forall p, q \in V \quad \mathcal{F}(k p, k q) \leq \mathscr{F}(h p, h q),
$$

where $\mathscr{F}(p, q):=\nu(p-q)$.

PROOF. There is $\lambda \in[0,1]$ such that $h=\lambda k$. Then

$$
\begin{gathered}
\mathcal{F}(k p, k q)=\nu(k p-k q)=\nu[k(p-q)] \leq \\
\leq \tau^{*}[\nu[\lambda k(p-q)], \nu[(1-\lambda) k(p-q)]] \\
\leq \tau^{*}\left[\nu[\lambda k(p-q)], \varepsilon_{0}\right]=\nu[\lambda k(p-q)]=\nu[h(p-q)]=\mathscr{F}(h p, h q) .
\end{gathered}
$$

THEOREM 3. Every $P N$-space $\left(V, \nu, \tau, \tau^{*}\right)$ has a completion, viz. is isometric to a dense linear subspace of a complete $P N$-space $\left(V^{\prime}, \nu^{\prime}, \tau, \tau^{*}\right)$.

PROOF. Exactly as in the proof of Theorem 1, one can prove that if both $p^{\prime}$ and $q^{\prime}$ belong to $V^{\prime}$, then $p^{\prime}+q^{\prime} \in V^{\prime}$. However, one can no longer use the same proof of the fact that if $\alpha \in \mathbb{R}$ and $p^{\prime} \in V^{\prime}$ then $\alpha p^{\prime} \in V^{\prime}$, because recourse was made to property (N2) which now may well not hold.

Now assume $\alpha \in \mathbb{R}$ and $p^{\prime} \in V^{\prime}$, let $\left\{p_{n}\right\} \in p^{\prime}$ and consider the sequence $\left\{\alpha p_{n}\right\}$. As a first step, we shall prove that it is a Cauchy sequence in $V$. This is obviously true for $\alpha=0$ and $\alpha=1$. Because of (N.2), it suffices to consider only the case $\alpha>0$. Now assume that $\left\{\alpha p_{n}\right\}$ is a Cauchy sequence for $\alpha=0,1, \cdots, k-1(k \in \mathbb{N})$. Then

$$
\begin{aligned}
\mathscr{F}\left(k p_{n}, k p_{m}\right) & =\nu\left[k\left(p_{n}-p_{m}\right)\right] \geq \tau\left[\nu\left(p_{n}-p_{m}\right), \nu\left[(k-1)\left(p_{n}-p_{m}\right)\right]\right]= \\
& =\tau\left[\mathcal{F}\left(p_{n}, p_{m}\right), \mathcal{F}\left((k-1) p_{n},(k-1) p_{m}\right)\right] .
\end{aligned}
$$

Since $\tau$ is continuous and

$$
\lim _{n, m \rightarrow \infty} \mathscr{F}\left(p_{n}, p_{m}\right)=\lim _{n, m-\infty} \mathscr{F}\left((k-1) p_{n},(k-1) p_{m}\right)=\varepsilon_{0}
$$


it follows that also $\left\{\alpha p_{n}\right\}$ is a Cauchy sequence for every $\alpha \in \mathbb{Z}_{+}$. If $\alpha$ is positive, but not integer, there exists $k \in \mathbf{Z}_{+}$such that $k<a<k+1$. Lemma 2 now gives

$$
\mathscr{F}\left((k+1) p_{n},(k+1) p_{m}\right) \leq \mathcal{F}\left(\alpha p_{n}, \alpha p_{m}\right) \leq \mathscr{F}\left(k p_{n}, k p_{m}\right) ;
$$

hence it is immediate to conclude that $\left\{\alpha p_{n}\right\}$ is a Cauchy sequence for every $\alpha \in \mathbb{R}_{+}$. Thus there exists an element $u^{\prime} \in V^{\prime}$ such that $\left\{\alpha p_{n}\right\} \in u^{\prime}$. Let us define $u^{\prime}=\alpha p^{\prime}$. In order to check that this is a good definition, let $\left\{p_{n}\right\} \sim\left\{p_{n}^{*}\right\}$. If $\alpha \in[0,1]$, it follows from Lemma 2 that $\mathcal{F}\left(p_{n}, p_{n}^{*}\right) \leq \mathcal{F}\left(\alpha p_{n}, \alpha p_{n}^{*}\right)$; since, by assumption $\mathscr{F}\left(p_{n}, p_{n}^{*}\right) \stackrel{w}{\rightarrow} \varepsilon_{0}$, also $\mathcal{F}\left(\alpha p_{n}, \alpha p_{n}^{*}\right) \stackrel{w}{\rightarrow} \varepsilon_{0}$. If $\alpha=k \in \mathbb{Z}_{+}$, as above, one has

$$
\begin{aligned}
\mathscr{F}\left(k p_{n}, k p_{n}^{*}\right) & =\nu\left[k\left(p_{n}-p_{n}^{*}\right)\right] \geq \tau\left[\nu\left(p_{n}-p_{n}^{*}\right), \nu\left[(k-1)\left(p_{n}-p_{n}^{*}\right)\right]\right]= \\
& =\tau\left[\mathcal{F}\left(p_{n}, p_{n}^{*}\right), \mathcal{F}\left((h-1) p_{n},(k-1) p_{n}^{*}\right)\right] .
\end{aligned}
$$

The same argument as above yields $\left\{k p_{n}\right\} \sim\left\{k p_{n}^{*}\right\}$ for every $k \in \mathbb{Z}_{+}$. Again, from this it is easy to obtain that, for every $\alpha \in \mathbb{R}$ one has $\left\{\alpha p_{n}\right\} \sim\left\{\alpha p_{n}^{*}\right\}$.

Therefore $V^{\prime}$ is a linear space. Only conditions (N.2) and (N.4) remain now to be proved. Proceeding as above, let $p^{\prime} \in V^{\prime}$ and let $\left\{p_{n}\right\}$ be a Cauchy sequence of elements of $V$ that belongs to $p^{\prime}$; then $\left\{-p_{n}\right\} \in-p^{\prime}$. Since $\nu^{\prime}$ is defined by (2.1), one has, on account of (N.2'), which holds for $\nu$,

$$
\nu^{\prime}\left(-p^{\prime}\right)=\lim _{n} \nu\left(-p_{n}\right)=\lim _{n} \nu\left(p_{n}\right)=\nu^{\prime}\left(p^{\prime}\right) .
$$

Moreover, for every $\alpha \in[0,1]$, one has, because $\tau^{*}$ is continuous,

$$
\begin{aligned}
\nu^{\prime}\left(p^{\prime}\right)=\lim _{n} \nu\left(p_{n}\right) & \leq \lim _{n} \tau^{*}\left[\nu\left(\alpha p_{n}\right), \nu\left((1-\alpha) p_{n}\right)\right] \\
& =\tau^{*}\left[\nu^{\prime}\left(\alpha p^{\prime}\right), \nu\left((1-\alpha) p^{\prime}\right)\right] .
\end{aligned}
$$

ACKNOWLEDGEMENT. The authors are grateful to their institutions for having made their cooperation possible. The third author is a member of the Italian G.N.A.F.A.-C.N.R.; he also gratefully acknowledges partial support from funds provided by the Italian M.U.R.S.T..

\section{REFERENCES}

1. ALSINA, C.; SCHWEIZER, B. and SKLAR, A., On the definition of a probabilistic normed space, Aequations Math. 46 (1993), 91-98.

2. MUŠTARI, D.H., The completion of random metric spaces, Kazan Gos. Univ. Učen. Zap. 127 (1967), kn.3, 109-119.

3. SCHWEIZER, B. and SKLAR, A., Probabilistic Metric Spaces, Elsevier North-Holland, New York, 1983.

4. SEMPI, C., On the space of distribution functions, Riv. Mat. Univ. Parma 4 (8) (1982), 243-250.

5. SEMPI, C., Hausdorff distance and the completion of probabilistic metric spaces, Boll. Un. Mat. Ital. 7 (6-B) (1992), 317-327.

6. S̆ERSTNEV, A.N., On the notion of a random normed space, Dokl. Akad. Nauk. SSSR 149 (2) (1963), 280-283.

7. SHERWOOD, H., On the completion of probabilistic metric spaces, Z. Wahrsch. Verw. Gebiete 6 (1966), 62-64.

8. SHERWOOD, H., Complete probabilistic metric spaces, Z. Wahrsch. Verw. Gebiete 20 (1971), 117-128.

9. SIBLEY, D.A., A metric for weak convergence of distribution functions, Rocky Mountain J. Math. 1 (1971), 427-430.

10. TAYLOR, M.D., New metrics for weak convergence of distribution functions, Stochastica 9 (1985), 5-17. 


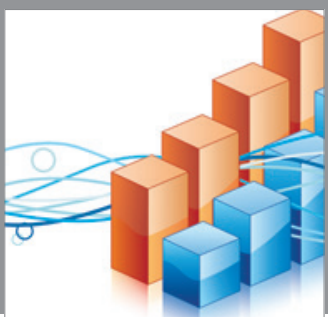

Advances in

Operations Research

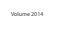

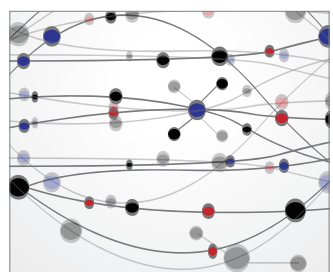

\section{The Scientific} World Journal
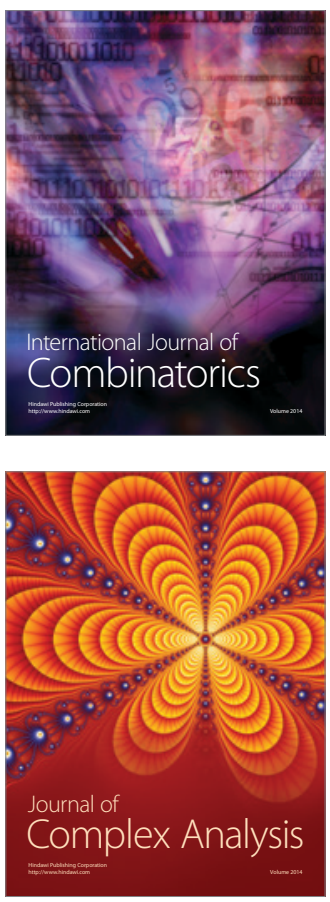

International Journal of

Mathematics and

Mathematical

Sciences
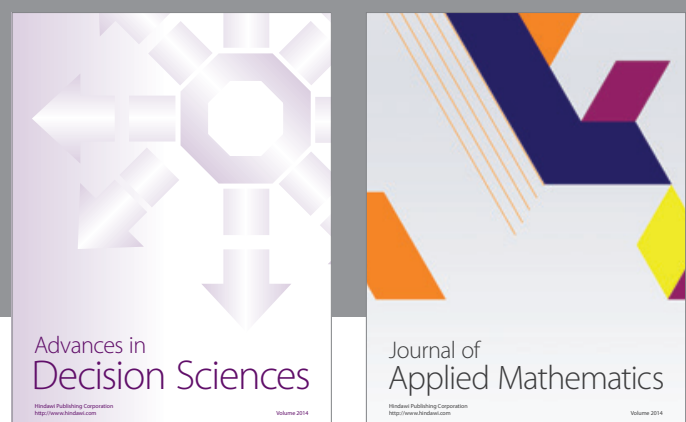

Journal of

Applied Mathematics
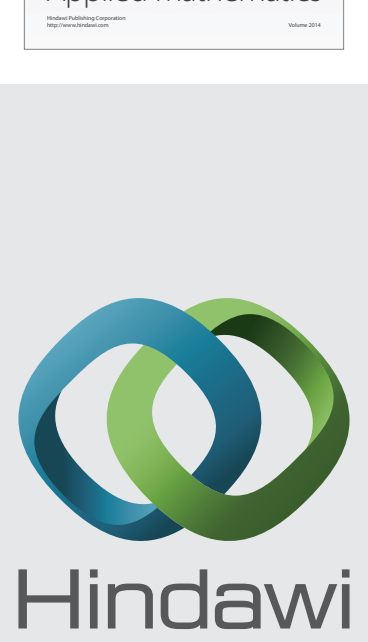

Submit your manuscripts at http://www.hindawi.com
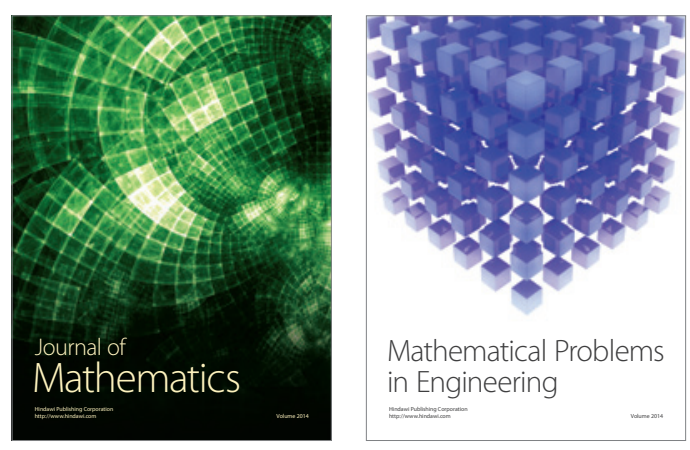

Mathematical Problems in Engineering
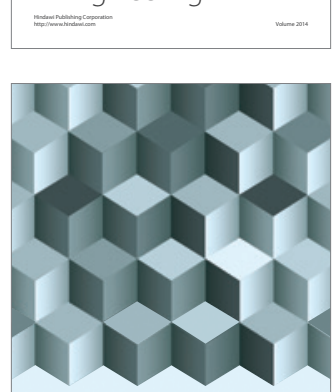

Journal of

Function Spaces
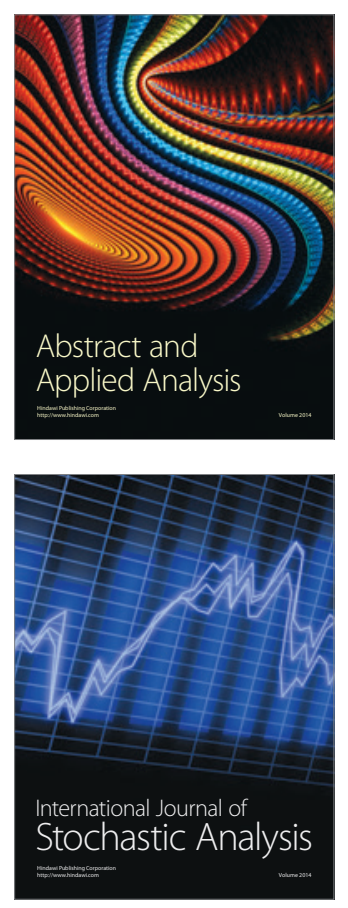

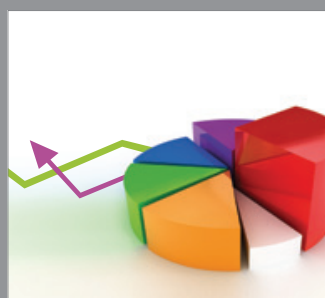

ournal of

Probability and Statistics

Promensencen
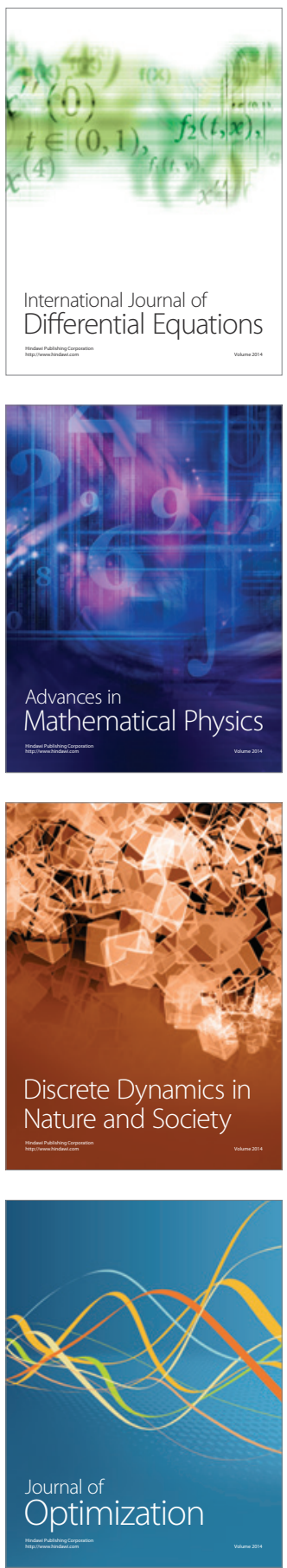